\title{
Bilateral Wilms tumors: Treatment results from a single center
}

\author{
Burça Aydın ${ }^{1}$, Canan Akyüz ${ }^{1}$, Bilgehan Yalçın ${ }^{1}$, Saniye Ekinci ${ }^{2}$, Berna Oğuz ${ }^{3}$, \\ Zuhal Akçören ${ }^{4}$, Ferah Yıldız ${ }^{5}$, Ali Varan ${ }^{1}$, Nilgün Kurucu ${ }^{1}$, Münevver Büyükpamukçu ${ }^{1}$, \\ Tezer Kutluk ${ }^{1}$ \\ ${ }^{1}$ Department of Pediatric Oncology, Hacettepe University Cancer Institute; Departments of ${ }^{2}$ Pediatric Surgery, ${ }^{3}$ Radiology, \\ ${ }^{4}$ Pathology and ${ }^{5}$ Radiation Oncology, Hacettepe University Faculty of Medicine, Ankara, Turkey. \\ E-mail: burcaaydin@yahoo.com
}

Received: 23rd May 2018, Revised: 5th July 2018, 20th July 2018, Accepted: 11th August 2018

SUMMARY: Aydın B, Akyüz C, Yalçın B, Ekinci S, Oğuz B, Akçören Z, Yıldız F, Varan A, Kurucu N, Büyükpamukçu M, Kutluk T. Bilateral Wilms tumors: Treatment results from a single center. Turk J Pediatr 2019; 61: 44-51.

The management of bilateral Wilms tumor (BWT) is challenging, particularly due to its presentation at a younger age, rarity, and difficulty for treatment decisions and surgical evaluation comparing to unilateral WT. In this study, the outcome of BWT patients from a single center who were treated by the Turkish Pediatric Oncology Group (TPOG) Wilms Tumor Regimen were retrospectively reviewed. From 1990 to 2016, 30 patients with synchronous BWT were treated with a preoperative chemotherapy of vincristine and actinomycin-D (VA). Chemotherapy was continued until safe nephron sparing surgery (NSS) could be performed for as long as radiological tumor response continued; otherwise, the chemotherapy was intensified by adding doxorubicin (D) alternating with VA every 6 weeks. The median followup of patients was 59 months (4-297 months). The median duration of preoperative chemotherapy was 81 days and ranged between 14 days and 198 days. Preoperative chemotherapy was modified in seven patients (23\%) to the VAD regimen. Twenty-two patients $(73 \%)$ had a radical nephrectomy on the larger tumor and NSS on the contralateral kidney, and 6 patients (20\%) had bilateral NSS. Postoperative tumor stages for stage I, II and III were $60 \%, 22 \%$ and $14 \%$, respectively. The 5 -year event free survival (EFS) rates were $100 \%, 90 \%$ and $51 \%$ for stages I, II and III $(p=0.02)$, respectively. Unfavorable histology and nephrogenic rests were reported in $20 \%$ and $20 \%$ of patients, respectively. The 5-year overall survival (OS) and EFS rates were $50 \%$ and $25 \%$, respectively, in patients with anaplasia, while the same rates were $96 \%$ and $96 \%$ in patients with favorable histology tumors $(\mathrm{p}=0.05$ and $\mathrm{p}<0.001$ ). The 10 -year EFS and OS rates for all patients were $82 \%$ and $86 \%$, respectively. Our results are comparable with the literature. VA is effective as initial preoperative treatment of BWT and allows for safe resection.

Key words: bilateral Wilms tumor, synchronous, preoperative chemotherapy, nephron sparing surgery.

Although the outcomes of unilateral Wilms tumors have greatly improved and few survivors experience serious late onset health issues, patients with bilateral Wilms tumors (BWT) have serious renal late effects from the treatments if renal tissue cannot be preserved $^{1-5}$; therefore, the treatment of BWT is still challenging. The most important concern is preserving renal function and leaving the maximum amount of normal renal parenchyma while maximally resecting the renal tumors to eliminate the risk of recurrence. Every patient needs tailored decisions on whether to intensify or prolong chemotherapy, as well as on the timing or type of surgery.

The outcome of patients with BWT has improved due to both preoperative chemotherapy and novel operative techniques in the preservation 
Table I. The TPOG Wilms Tumor Regimen.

Preoperative chemotherapy in Wilms tumor

V $1.5 \mathrm{mg} / \mathrm{m}^{2}$ (max. $2 \mathrm{mg}$ ), once weekly for 4 weeks + A $15 \mu \mathrm{g} / \mathrm{kg} /$ day (5 days)

Postoperative chemotherapy in Wilms tumor according to disease stages and histopathology

Stage I FH and UH, IIA FH patients

$\mathrm{V} 1.5 \mathrm{mg} / \mathrm{m}^{2}$ (max. $2 \mathrm{mg}$ ), once weekly + A $15 \mu \mathrm{g} / \mathrm{kg} /$ day (5 days), every 6 weeks for a total of 5 cycles in 6 months

Stage IIB FH

$\mathrm{V} 1.5 \mathrm{mg} / \mathrm{m}^{2}$ (max. $2 \mathrm{mg}$ ), once weekly and A $15 \mu \mathrm{g} / \mathrm{kg} /$ day (5 days), every 6 weeks for a total of

12 months and external radiotherapy to tumor bed

Stage III and IV FH

$\mathrm{V} 1.5 \mathrm{mg} / \mathrm{m}^{2}$ (max. $2 \mathrm{mg}$ ), once weekly; A $15 \mu \mathrm{g} / \mathrm{kg} /$ day (5 days), every 6 weeks; alternating doxorubicin $20 \mathrm{mg} / \mathrm{m}^{2} /$ day (3 days), every 6 weeks; for a total of 12 months and external radiotherapy to abdomen

Stages II, III, and IV UH tumors

V $1.5 \mathrm{mg} / \mathrm{m}^{2}$ (max. $2 \mathrm{mg}$ ), once weekly, A $15 \mu \mathrm{g} / \mathrm{kg} /$ day (5 days), every 6 weeks; alternating doxorubicin $20 \mathrm{mg} / \mathrm{m}^{2} /$ day (3 days), every 6 weeks; and etoposide $100 \mathrm{mg} / \mathrm{m}^{2} /$ day (3 days), every 6 weeks; for a total of 18 months and external radiotherapy to abdomen

Doxorubicin was discontinued after reaching allowed cumulative doses.

A: actinomycin-D, D: doxorubicin, FH: favorable histology, UH: unfavorable histology, V: vincristine.

of renal parenchyma. The results from international and national study groups have helped to draw some guidelines for this specific group of patients. ${ }^{6-12}$ The Turkish Pediatric Oncology Group (TPOG) has been conducting a study on renal tumors since 1998 based on the principles of the SIOP-93 regimen. The TPOG group reported the treatment results from unilateral WTs ${ }^{13}$ and the outcomes of surgery for BWT. ${ }^{14}$ In this study, the outcome of BWT patients from a single center who were treated by the TPOG Wilms tumor regimen were retrospectively reviewed.

\section{Material and Methods}

From January 1990 to July 2016, 303 patients with Wilms tumors were treated in our center. Among this group, 30 consecutive patients with synchronous BWT were treated. The hospital files and medical records for these patients were retrospectively reviewed and evaluated for this study. All patients were evaluated with at least two different radiological examinations of the abdomen: with ultrasound (US) and either computed tomography (CT) or magnetic resonance imaging (MR). The metastatic evaluation was primarily based on posterior, anterior and lateral chest X-rays, but thoracic CT was added to the metastatic workup if visible lung metastases were detected.
All patients were discussed by an institutional multidisciplinary tumor board consisting of pediatric oncologists, pediatric surgeons, pediatric pathologists, pediatric radiologists and pediatric radiation oncologists; the board first discussed the initial radiological findings for staging and later discussed evaluation of the response to preoperative treatment and surgical interventions using additional radiological findings.

Preoperative chemotherapy consisted of vincristine and actinomycin-D (VA). The details of the regimen are given in Table I. Chemotherapy consisted of vincristine (V) (1.5 $\mathrm{mg} / \mathrm{m}^{2}$, D1 and then weekly for 4 weeks) and actinomycin-D (A) $(15 \mu \mathrm{g} / \mathrm{kg} /$ day, D1, D2, D3, D4 and D5). At the $5^{\text {th }}$ week, abdominal US and CT scans were performed to evaluate the patients' response, then each patient's case was discussed by the tumor board in preparation for the initial surgery. If the tumors responded and the tumor volume decreased, but nephronsparing surgery (NSS) was not possible, a second cycle of the VA regimen was given, and a radiological evaluation was repeated at the end of it. At the end of the second cycle, the patients were discussed by the tumor board to decide on surgery strategies. If the tumors regressed and bilateral NSS were possible, patients underwent surgery, and further treatment was planned 
with postoperative staging and histopathological findings (Table I). Otherwise, preoperative chemotherapy was continued, depending on the response, either with the VA regimen (if tumor volume continued to decrease) or the VAD regimen (if the tumor was stable), which was intensified by adding doxorubicin (D) (20 $\mathrm{mg} / \mathrm{m}^{2}, \mathrm{D} 1, \mathrm{D} 2$ and D3 alternating with VA in every 6 weeks). Chemotherapy was continued until a safe NSS could be performed, as long as the tumor continued to respond to radiological intervention.

If possible, surgery was performed on both kidneys during the same operation, and the goal was bilateral NSS. Unless it was necessary, bilateral radical nephrectomies (RN) were avoided in all patients. NSS was defined as a partial nephrectomy with negative tumor margins and enucleation of the tumor. A unilateral $\mathrm{RN}$ was performed if the kidney had little tumor-free parenchyma. Biopsy or tumor resection were considered for tumors that showed no response to chemotherapy after intensification. Additional radiological tests were performed to show the renal anatomy and vascular structures for precise surgery if needed.

All the patients who survived periodically followed up to check renal function. Blood pressure, serum creatinine and BUN levels, urine glomerular filtration rates (GFR), and urinary protein excretion were checked in routine controls.

The time intervals for survival parameters started at the time of admission and were measured to the date of relapse, to progression of event free survival (EFS), to death, until the last contact, or when the patient met the study endpoint of overall survival (OS). OS and EFS were calculated using the KaplanMeier method. The comparisons between groups for different variables were drawn with a log-rank test. A p value $<0.05$ was considered statistically significant for each test. The statistical analysis was performed using SPSS v20.0. Approval for the study was granted by the Ethics Committee of Hacettepe University (Decision no: 16969557/444, dated: 29.03.2017).

\section{Results}

There were 30 patients with synchronous bilateral tumors among the 303 patients treated for Wilms tumors, making the prevalence rate $9.9 \%$. The median age of the patients was 21.7 months and the F/M ratio was 1.3. There were two patients with WAGR syndrome and one with hemihypertrophy. These patients were diagnosed during routine follow-up. One patient with WAGR syndrome had bilateral stage I tumors and other two patients had stage I tumors in one kidney and stage II and III tumors in contralateral kidneys after surgery. Three patients had lung metastases, one patient had unilateral renal vein thrombosis and one had intra-abdominal lymph node metastasis on admission. The clinical characteristics of the patients are shown in Table II.

The median follow-up of patients was 59 months (range: 4-297 months); all patients were followed for an average of 46 months (range: 4-297 months). The median duration of preoperative chemotherapy was 81 days (14-198 days). One patient underwent early surgery due to spontaneous tumor rupture noted on the CT images performed at the time of diagnosis. She only received two weeks of preoperative chemotherapy. Total chemotherapy duration was median 307 days (204-652). Twenty-seven patients received VA as preoperative chemotherapy. Three patients received the VAD regimen due to local advanced disease. Chemotherapy had to be modified in seven patients $(23 \%)$ after 4 weeks of preoperative treatment due to stable disease. After resection, three of these seven patients were found to have unfavorable histology (UH) tumors, and another four had cystic partially differentiated nephroblastoma or epithelial tumors. Eleven patients needed intensification of chemotherapy after surgery. Ten patients completed chemotherapy with VA only, and they have been well with no evidence of disease.

Twenty-two patients had an RN on the kidney with the larger tumor and NSS on the contralateral kidney, and 6 patients $(20 \%)$ had bilateral NSS. Two of the six patients who had bilateral NSS had their surgeries performed in two separate sessions, and four had NSS in one session. Types of surgeries are summarized in Table III. Six patients underwent two procedures for tumor resection. Three of them had an RN in the first session then NSS in the second operation (one partial nephrectomy and 
Table II. Patient Characteristics.

\begin{tabular}{lc}
\hline Characteristics & Results, N (\%) \\
\hline Sex & $13(43.3)$ \\
Male & $17(56.7)$ \\
Female & $21.7(3-75)$ \\
Age, months* & \\
Genetic malformations/syndromes & 2 \\
$\quad$ WAGR syndrome & 1 \\
Hemihypertrophy & 1 \\
Horseshoe kidney & 2 \\
VUR reflux and hydronephrosis & 1 \\
Hypertensive cardiomyopathy & \\
Histology & $6(20.0)$ \\
Nephrogenic rest & $24(80.0)$ \\
Favorable histology & $6(20.0)$ \\
Unfavorable histology & \\
Metastases & $3(10.0)$ \\
Lung & $1(3.3)$ \\
Thrombi in vena cava & $1(3.3)$ \\
Distant lymph node metastases & \\
Higher local stage (29 patients) & $11(37.9)$ \\
I & $10(34.5)$ \\
II & $8(27.6)$ \\
III & $37(63.8)$ \\
Postoperative Stage (each kidney; N=58) & $13(22.4)$ \\
I & $9(15.5)$ \\
II & \\
\hline & \\
\hline
\end{tabular}

*: Values are is presented as median (minimum-maximum)

A: actinomycin-D; D: doxorubicin; NSS: nephron-sparing surgery; RN: radical nephrectomy; V: vincristine; VUR: vesicoureteral reflux; WAGR: Wilms tumor, aniridia, genitourinary anomalies, and mental retardation.

two enucleation). These three patients are still followed with no evidence of disease at 262 , 25 and 29 months, respectively. Two patients had a partial nephrectomy and enucleation at the first session and a partial nephrectomy and enucleation at second session; they are well with no relapse at 297 and 52 months of follow-up. One patient had bilateral renal biopsies. This patient had bilateral RN for bilateral anaplasia noted on the tumor biopsies, and he died of renal failure with underlying cardiomyopathy and heart failure.

Four patients had no surgery on the contralateral kidney since the tumors had disappeared on their preoperative radiological images and inspection during surgery confirmed the radiological findings. These patients have been relapse-free for 42, 117, 270 and 297 months. In another two patients, the intraoperative biopsies of residual lesions showed no tumor. These tumors were staged as stage I tumors, and further treatment was planned based on other renal tumors if they had higher stage of tumor.

The local stages of the 58 tumors were $60 \%$, $22 \%$ and $14 \%$ for stage I, II and III, respectively. The larger tumors' stages for each patient were $38 \%, 34 \%$ and $28 \%$ for stage I, II and III, respectively. Overall, 34 kidneys were spared from $\mathrm{RN}(67 \%)$. A total of $57 \%$ of patients 
Table III. Treatment Characteristics of the Study Group

\begin{tabular}{|c|c|}
\hline Treatment & $\mathrm{N}(\%)$ \\
\hline Preoperative radiotherapy & $4(13.3)$ \\
\hline Postoperative radiotherapy & $6(20.0)$ \\
\hline \multicolumn{2}{|l|}{ Definitive surgery } \\
\hline \multirow{4}{*}{ RN with contralateral biopsy } & $22(73.3)$ \\
\hline & $3 / 22$ \\
\hline & $2 / 22$ \\
\hline & $6(20.0)$ \\
\hline $\begin{array}{l}\text { - } \quad \text { Bilateral NSS } \\
\text { NSS with contralateral inspection }\end{array}$ & $1 / 6$ \\
\hline \multirow{2}{*}{$\begin{array}{l}\text { - Bilateral needle biopsies } \\
\text { Bilateral nephrectomy following biopsy }\end{array}$} & $2(6.7)$ \\
\hline & $1 / 2$ \\
\hline Total & $30(100.0)$ \\
\hline
\end{tabular}

NSS: nephron sparing surgery, RN: radical nephrectomy

underwent surgery in 3 months.

Four patients received local radiotherapy (RT) before surgery due to unresectable or progressive tumors or persistent thrombi in the vena cava, despite several modifications of the chemotherapy. Radiotherapy doses ranged between 900 and 1800 cGy. Preoperative RT allowed NSS in one of their kidneys in two patients. Six more patients received RT after surgery for locally advanced tumors. Negative tumor margins could not be obtained after enucleation of multiple tumors in three kidneys of two different patients. Postoperative chemotherapy was intensified with the VAD regimen and no RT was planned, although one showed focal anaplasia. Both are well with no sign of relapse on follow-up at 33 and 219 months. Six patients received local RT for stage III tumors, but two patients with stage III tumors with epithelial histology did not receive RT and no local relapse was developed.

Histopathological examination showed unfavorable histology (UH) in eight tumors from six patients $(20 \%)$. Anaplasia was diagnosed in seven tumors from six patients, and blastemal histology was found in one tumor. Nephrogenic rests were reported in six patients $(20 \%)$. The 5-year OS and EFS rates were $50 \%$ and $25 \%$, respectively, in patients with anaplasia, while the same rates were $96 \%$ and $96 \%$ in patients with $\mathrm{FH}$ tumors $(\mathrm{p}=0.05$ and $\mathrm{p}<0.001)$. Survival rates are shown in Figure 1a. Three patients with unresponsive tumors showed anaplasia, another four had cystic partially differentiated nephroblastoma or epithelial Wilms tumors.

The 10-year OS and EFS rates for all patients were $86 \%$ and $82 \%$, respectively (Fig. 1b). The 5 -year EFS rates for patients with stages I, II and III were $100 \%, 90 \%$ and $51 \%$, respectively $(p=0.02)$ (Fig. 1c). The 5-year OS rates were $100 \%, 90 \%$ and $63 \%$ for stages I, II and III, respectively $(p=0.08)$. Only one patient died immediately after surgery due to cardiac and renal failure. This patient had bilateral anaplasia at initial needle biopsies and had to undergo bilateral RN and dialysis. She previously had hypertensive cardiomyopathy and died shortly after surgery. None of the patients who survived currently have renal failure. All patients had a GFR $>60 \mathrm{~mL} / \mathrm{min} / 1.73 \mathrm{~m}^{2}$ at last follow-up.

\section{Discussion}

Synchronous bilateral Wilms tumors need special consideration when treating them. International collaborative studies have been shaping the principles of treatment, and the outcome has greatly improved over the last few decades. ${ }^{6-10,11,15}$ It is critical that these principles and results be reproducible in other centers. Therefore, we presented the treatment and results of BWT in our center. The long-term 

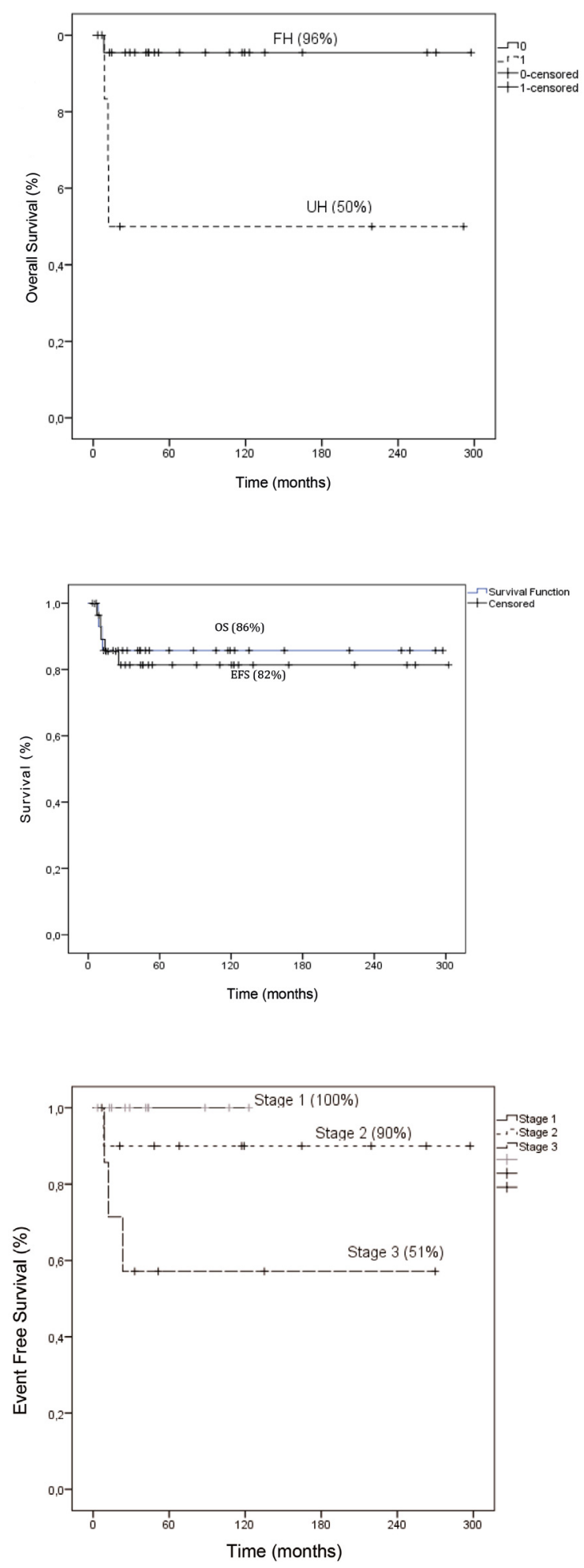

Fig. 1. a) Overall survival rates for unfavorable (UH) and favorable histology (FH) tumors

b) Event free (EFS) and overall survival (OS) rates of patients

c) Event free survival rates for stages I, II and III
EFS of $82 \%$ and OS of $86 \%$ were analogous with other single or multicenter groups. The only discrepancy in this series of patients with BWT was the higher prevalence rate of $9.9 \%$, even compared to the previously reported results from TPOG. $7,8,10-13$ Our center is one of the largest and most experienced pediatric cancer centers, and the higher prevalence might be a reflection of the high referral rate of difficult cases. Other demographic features such as gender, median age, UH or genetic syndromes were consistent with literature. Nephrogenic rests were found in 6 children, higher than expected for unilateral tumors $(20 \%)$. Unfavorable histology in at least one kidney was detected in 6 of the children (20\%). $\mathrm{UH}$ was a poor prognostic factor as reported in other studies. ${ }^{10-12,15,16}$ The 5-year OS and EFS rates in patients with UH were $50 \%$ and $25 \%$, respectively, which is worse than patients with $\mathrm{FH}$ tumors.

A median preoperative chemotherapy duration of 11.6 weeks produced $64 \%$ of stage I tumors. Although some patients needed as long as 30 weeks to have a safe resection, $57 \%$ of the children had tumor removal within 3 months. Bilateral NSS were performed in 6 patients $(20 \%)$. Some studies suggest that 12 weeks is the optimum duration for preoperative chemotherapy to allow for a complete and safe NSS. ${ }^{10,11}$ Although NSS leaves functioning renal parenchyma, there is always the risk of leaving positive tumor margins, especially for the multifocal tumors. The major concern with microscopic residual tumors is the risk of local or distant relapses and chemotherapy intensification and/or RT is then needed for the local control. There are reports that microscopic residue might not increase the recurrence rate. ${ }^{17}$ In this series of patients, there were 3 kidneys with positive microscopic margins in our group, and RT was not given due to multiple tumors. All three tumors had favorable histology and intensification of chemotherapy seemed to control the residual microscopic disease. In addition, 6 tumors disappeared completely with preoperative chemotherapy, and all have been well and off-therapy, with no evidence of tumor. Our experience supports this comment. However, evidence is scarce and microscopic residue after surgery should be discussed cautiously. 
The outcome and treatment success has tremendously improved for WT over the past few decades, and BWT followed this improvement. The SIOP studies showed that preoperative chemotherapy enabled the surgeons to perform safe surgeries and preserve the maximum amount of renal parenchyma. Preoperative chemotherapy has been the backbone in the treatment of BWT since then. There are differences in the contents and duration of preoperative regimens. SIOP and SIOP-like regimens consist of two drugs: vincristine and actinomycin. ${ }^{11}$ There are reports doxorubicin was added to VA (VAD) as initial treatment in selected cases. ${ }^{10}$ The AEIOP group reported that the VAD regimen seemed to produce a better local control rate. However, VA and VAD produced similar treatment outcomes and the outcome difference was statistically insignificant. ${ }^{10}$ The physician chose whether to use the VAD regimen; the decision was not made based on previously determined criteria or the presence of high-risk features of the tumors. Therefore, it is not easy to conclude whether a more intensive regimen warrants better outcomes.

In our series of patients, 10/30 patients received treatment with VA only, and all were long-term survivors, suggesting not all patients with BWT need an intensive regimen upfront. It should also be considered that some patients did not need doxorubicin at all. If a preoperative VAD regimen enables better local control, this should be reflected in the survival gain.

The only limitation of this study is its retrospective manner. Although patients were intended to be treated from the time of diagnosis, the evaluation had to be done retrospectively due to its small numbers. Quite a number of published studies are done retrospectively due to the small number of patients with BWT and because their treatments are heterogeneous, even in the same center. Ongoing prospective studies with cumulative results from centers will address the effect of a more intensified preoperative chemotherapy on outcomes.

\section{REFERENCES}

1. Breslow NE, Collins AJ, Ritchey ML, Grigoriev YA, Peterson SM, Green DM. End stage renal disease in patients with Wilms tumor: Results from the National Wilms Tumor Study Group and the United States Renal Data System. J Urol 2005; 174: 1972-1975.

2. Davidoff AM, Interiano RB, Wynn L, et al. Overall survival and renal function of patients with synchronous bilateral Wilms tumor undergoing surgery at a single institution. Ann Surg 2015; 262: 570-576.

3. Aronson DC, Slaar A, Heinen RC, de Kraker J, Heij HA. Long-term outcome of bilateral Wilms tumors (BWT). Pediatr Blood Cancer 2011; 56: 1110-1113.

4. Grigoriev Y, Lange J, Peterson SM, et al. Treatments and outcomes for end-stage renal disease following Wilms tumor. Pediatr Nephrol 2012; 27: 1325-1333.

5. Giel DW, Williams MA, Jones DP, Davidoff AM, Dome JS. Renal function outcomes in patients treated with nephron sparing surgery for bilateral Wilms tumor. J Urol 2007; 178 (4 Pt 2): 1786-1789.

6. Hamilton TE, Ritchey ML, Argani P, et al. Synchronous bilateral Wilm's tumor with complete radiographic response managed without surgical resection: A report from the National Wilm's Tumor Study 4. J Pediatr Surg 2008; 43: 1982-1984.

7. Hamilton TE, Ritchey ML, Haase GM, et al. The management of synchronous bilateral Wilms tumor: A report from the National Wilms Tumor Study Group. Ann Surg 2011; 253: 1004-1010.

8. Oue T, Koshinaga T, Okita H, Kaneko Y, Hinotsu S, Fukuzawa M. Bilateral Wilms tumors treated according to the Japan Wilms Tumor Study Group protocol. Pediatr Blood Cancer 2014; 61: 1184-1189.

9. Horwitz JR, Ritchey ML, Moksness J, et al. Renal salvage procedures in patients with synchronous bilateral Wilms' tumors: A report from the National Wilms Tumor Study Group. J Pediatr Surg 1996; 31: 1020-1025.

10. Indolfi $P$, Jenkner $A$, Terenziani $M$, et al; AIEOP Wilms Tumor Working Group. Synchronous bilateral Wilms tumor: A report from the Associazione Italiana Ematologia Oncologia Pediatrica (AIEOP). Cancer 2013; 119: 1586-1592.

11. Sudour H, Audry G, Schleimacher G, Patte C, Dussart S, Bergeron C. Bilateral Wilms tumors (WT) treated with the SIOP 93 protocol in France: Epidemiological survey and patient outcome. Pediatr Blood Cancer 2012; 59: 57-61.

12. Chen SH, Hung IJ, Yang CP, et al. Clinical features and long-term outcomes of bilateral Wilms tumor treated with Taiwan Pediatric Oncology Group protocols: A single center report. Asia Pac J Clin Oncol 2016; 12: 300-307.

13. Sarhan OM, El-Baz M, Sarhan MM, Ghali AM, Ghoneim MA. Bilateral Wilms tumors: Single-center experience with 22 cases and literature review. Urology 2010; 76: $946-951$. 
14. Akyüz C, Yalcin B, Yildiz I, et al. Treatment of Wilms tumor: A report from the Turkish Pediatric Oncology Group (TPOG). Pediatr Hematol Oncol 2010; 27: 161-178.

15. User IR, Ekinci S, Kale G, et al. Management of bilateral Wilms tumor over three decades: The perspective of a single center. J Pediatr Urol 2015; 11: 118.e1-6.

16. Hamilton TE, Green DM, Perlman EJ, et al. Bilateral Wilms tumor with anaplasia: Lessons from the National Wilms Tumor Study. J Pediatr Surg 2006; 41: 1641-1644.
17. Shamberger RC, Haase GM, Argani P, et al. Bilateral Wilms tumors with progressive or nonresponsive disease. J Pediatr Surg 2006; 41: 652-657.

18. Kieran K, Williams MA, Dome JS, McGregor LM, Krasin MJ, Davidoff AM. Margin status and tumor recurrence after nephron-sparing surgery for bilateral Wilms tumor. J Pediatr Surg 2013; 48: 1481-1485. 